\title{
Epidemiology, species distribution, antifungal susceptibility and outcome of candidemia among Internal Medicine Wards of community hospitals of Udine province, Italy
}

\author{
Federico Silvestri, ${ }^{1}$ Matteo Bassetti, ${ }^{2}$ Marco Zaramella, ${ }^{1}$ Liana Domenis, ${ }^{3}$ Vito Di Piazza, ${ }^{3}$ Massimiliano Fazio, ${ }^{4}$ \\ Mario Maschio, ${ }^{4}$ Valentino Moretti ${ }^{5}$ for FADOI-FVG \\ ${ }^{1}$ Division of Internal Medicine, General Hospital, Latisana (UD); ${ }^{2}$ Infectious Diseases Division, Santa Maria Misericordia \\ University Hospital, Udine; ${ }^{3}$ Division of Internal Medicine, General Hospital, Tolmezzo (UD); ${ }^{4}$ Division of Internal Medicine, \\ General Hospital, Palmanova (UD); ${ }^{5}$ Division of Internal Medicine, General Hospital, San Daniele del Friuli (UD), Italy
}

\begin{abstract}
Candidemia is an emerging problem among patients hospitalized in Internal Medicine Wards (IMW). We performed a retrospective study to assess the epidemiology, species distribution, antifungal susceptibility and outcome of candidaemia recorded over a 3-year period (2010-2012) among IMW of community hospitals of Udine province in Italy: forty-eight patients were identified, with an overall incidence of 1.44 cases/1000 hospital admissions/year. Candida albicans was the most frequent species, followed by Candida parapsilosis that accounted for $42.9 \%$ of Tolmezzo cases. All isolates were susceptible to amphotericin and caspofungin, while $11.4 \%$ of strains were not-susceptible to voriconazole and $14.3 \%$ to fluconazole. Crude mortality was $41.7 \%$. In conclusion, in community hospitals overall incidence of candidemia is similar to tertiary care hospitals, but $80 \%$ of cases are detected in IMW. Candida species distribution is overlapping, but differences in local epidemiology were found and should be taken into consideration. No resistance to amphotericin and caspofungin was found while resistance to azoles was observed. Knowledge of this data might be useful when planning the best therapeutic strategy.
\end{abstract}

Correspondence: Federico Silvestri, Divisione di Medicina Interna, Ospedale Civile, via Sabbionera 45, 33053 Latisana (UD), Italy.

Tel.: +39.0431.529341 - Fax: +39.0431.529467.

E-mail: silvestrifederico@alice.it

Key words: candidemia, internal medicine wards, community hospital.

Contributions: FS data analyzing and manuscript writing; MB manuscript rewiewing and references search; MZ, LD, VDP, MF, MM and VM clinical data collecting. MR, FP, NB, SM, FP, SDM and FS (listed in the appendix) microbiological data collecting.

Conflict of interests: MB serves on scientific advisory boards for Pfizer Inc., MSD, Gilead and Astellas Pharma Inc.; has received funding for travel or speaker honoraria from the same companies (Pfizer Inc., MSD, Gilead Sciences and Astellas Pharma Inc.). All other authors declare no potential conflict of interests.

Conference presentation: part of this paper was presented at the XVII Italian Meeting FADOI, 2013 May 11-14, Giardini Naxos (ME), Italy

Received for publication: 5 February 2014.

Accepted for publication: 6 April 2014

This work is licensed under a Creative Commons Attribution NonCommercial 3.0 License (CC BY-NC 3.0).

(C) Copyright F. Silvestri et al., 2014

Licensee PAGEPress, Italy

Italian Journal of Medicine 2014: 8:253-258

doi:10.4081/itjm.2014.481

\section{Introduction}

In recent years the epidemiology of candidemia has been extensively studied in the United States, Europe and some South America countries and increasing evidence suggests that Candida is a growing and underestimated cause of bloodstream infection (BSI). ${ }^{1}$

Infact candidemia episodes raised fivefolds in the past ten years with a current incidence of $\geq 1 / 1000$ hospital admissions/year and Candida spp. is currently between the fourth and the sixth most common nosocomial bloodstream isolate in American and European studies. ${ }^{2,3}$

Candidemia has also been associated with high rates of crude (as high as $81 \%$ ) and attributable (from 25 to $71 \%$ ) mortality and with increased healthcare expenses, due to prolongation of hospitalization and drugs cost. ${ }^{4-10}$

Moreover, in terms of Candida species, a shift towards non-albicans species was recently reported, especially in some hospital settings, ${ }^{11-13}$ with increasing resistance to azoles also being underlined. ${ }^{5,14}$

Nevertheless, the majority of studies were performed in tertiary care hospitals where most of the Candida BSI are detected in intensive care units and solid organ transplant or hematological wards, with only few recent papers focusing also on internal medicine wards (IMW). ${ }^{1,15-17}$

The complete lack of data on candidemias in IMW 
of community hospitals, prompted us to perform the present retrospective study on epidemiology, species distribution, antifungal susceptibility and outcome of Candida spp. BSI among primary care hospitals of Udine province.

\section{Materials and Methods}

The study was carried out retrospectively in the IMW of all five community hospitals (Latisana, Palmanova, San Daniele del Friuli, Tolmezzo and Gemona) of area vasta udinese, located in North-east Italy and covering Udine province with a catchment area of around 300,000 inhabitants.

During the study period (January 2010 - December 2012) a total of 33,257 admissions, evenly distributed among years (around 11,000/year) and hospitals (between 1900 and 2700/hospital/year) were observed in the five IMW.

All patients who developed at least one positive blood culture for Candida spp. in the study period were included. Only the first episode of candidemia was recorded.

Patients were identified through the microbiological laboratory database of 3 laboratories (one serving Latisana and Palmanova hospitals, one serving San Daniele hospital and the last serving Tolmezzo and Gemona hospitals); the specific fungal pathogen and antifungal susceptibility tests were then analyzed and clinical data were collected consulting patients hospital charts.

Outcome was recorded until death or hospital discharge.

Candida species were isolated from blood using the BAC-TEC 860 system (Becton Dickinson, Inc., Sparks, MD, USA) in 3 hospitals and the BacT/ALERT system (bioMerieux, Inc., St. Louis, MO, USA) in the other two.

The species were identified using API ID 32C system (bioMerieux), MicroScan WalkAway system (Siemens Healthcare Diagnostics, Erlangen Germany) or Vitek 2 system (bioMerieux) and antifungal susceptibility was performed using Sensitre YeastOne Y-8 panel (Trek Diagnostic Systems, Cleveland, OH, USA) or Vitek2 fungal susceptibility card AST-YS01 (bioMerieux).

The interpretative breakpoints were based on values recommended by the Clinical and Laboratory Standards Institute (CLSI) and, from March 2012, by the European Committee on Antimicrobial Susceptibility Testing (EUCAST).

\section{Results}

A total of 48 patients with at least one episode of candidemia were identified during the study period in the IMW of the five hospitals: 19 cases in 2010, 17 in
2011 and 12 in 2012, with only one Medicine (Tolmezzo) showing a progressive increase in the number of cases (2, 4 and 8 cases in the three years) and no cases found in Gemona. Corresponding overall incidence was 1.44 cases/1000 admissions (1.70 in 2010; 1.55 in 2011 and 1.08 in 2012). In the same period, other 12 patients with candidemia were detected in different wards of the same hospitals (6 in Emergency wards and 6 in Surgery departments), for a total of 60 cases.

Of 48 identified patients, $29(60.4 \%)$ were male and $19(39.6 \%)$ female; mean age was $71.1 \pm 16.7$ years and median was 76.0 (22-97) years, with 36 patients (75.0\%) older than 65 years and $26(54.2 \%)$ older than 75 , and with Tolmezzo patients being younger (mean $58.3 \pm 33.2$, median 66.0 , range 22-87) than in other hospitals.

Thirty-six patients $(75.0 \%)$ were admitted to IMW coming from home, while $10(20.8 \%)$ were transferred from other hospital wards and only 2 (4.2\%) were coming from long-care facilities.

Large majority of patients (43-89.6\%) were febrile at the time of positive blood culture.

Crude mortality, during hospital stay, was $41.7 \%$ (20/48).

Table 1 shows main risk factors associated with candidemia: prior/current antibiotic (in our population a mean of $12.5 \pm$ days before positive blood culture) and corticosteroid therapy, older age, male sex, some comorbidities and any kind of invasive devices (parenteral nutrition, central venous catheter, urinary catheter, dialysis, etc.) were found to be frequently associated with candidemia.

The distribution of isolated Candida species is shown in Table 2. Overall, Candida albicans caused $60.4 \%$ of cases and was the most frequent strain in all hospitals, followed by Candida parapsilosis and tropicalis, with only few cases of Candida glabrata and krusei found. Notably, C. parapsilosis accounted for $42.9 \%$ of cases of Tolmezzo Medicine.

Table 3 shows the results of the susceptibility tests performed. The rate of resistance to amphotericin B and caspofungin was 0 for all strains; for $C$. albicans it was $10.5 \%$ to fluconazole and $15.8 \%$ to voriconazole; for C. glabrata it was $66.7 \%$ to fluconazole and $33.3 \%$ to voriconazole; the only Candida krusei was resistant to fluconazole, while all strains of $C$. parapsilosis and C. tropicalis were sensitive to all antifungal agent tested. Overall resistance to azoles was $14.3 \%$ to fluconazole and $11.4 \%$ to voriconazole.

Resistant strains were equally distributed among hospitals ( 1 in Palmanova, 2 each in the other 3 hospitals).

Distribution of Candida spp. in wards other than Medicine was, overall, as follows: $\mathrm{N}=5$ C. albicans, $\mathrm{N}=4$ C. parapsilosis, $\mathrm{N}=2$ C. glabrata and $\mathrm{N}=1$ Candida catenulata. 
In 8 cases $(16.7 \%)$ a bacterial co-infection was also found (4 cases of coagulase-negative Staphylococci, 1 Streptococcus mitis, 1 Propinibacterium acnes, 1 Escherichia coli and 1 Pseudomonas aeruginosa + Enterococcus faecium).

\section{Discussion and Conclusions}

Although IMW represent a significant reservoir of patients with candidemia, with a prevalence ranging from 24 to $57 \%$, only few investigators have specifically addressed the epidemiological aspects of candidemia among internal medicine patients, ${ }^{1,15-17}$ and, above all, no studies have been performed in community hospitals.

Our study shows that the cumulative incidence of candidemia among five community hospitals of northeast Italy over a 3-year observation period (1.44 cases/1000 admissions), was overlapping that of five tertiary care hospitals recently described in Italy and Spain, ${ }^{1}$ with the major difference being the fact that among our community hospitals the great majority of cases $(80.0 \%)$ were detected in IMW. The lack of intensive care units and solid organ transplant or hematological wards in this hospital setting may explain this difference.

Observation of demographic characteristics of our patients and of risk factors associated with candidemia, showed that older age $(54.2 \%$ of patients older than 75 years, as expected in IMW), prior/current antibiotic and corticosteroid therapy, the presence of some comorbidities (in particular solid tumors, diabetes mellitus, previous surgery) and any kind of indwelling devices (parenteral nutrition, central venous catheter, urinary catheter, dialysis, etc.), were frequently associated with candidemia. The risk factors herein described are overlapping those already described in the Internal Medicine setting, ${ }^{1,16}$ with the major difference being again, in our population, the lack of association with hematologic malignancies, solid organ transplant, HIV and other diseases typically referred to tertiary care hospitals.
On the other hand, the association of candidemia with cardiovascular diseases, described also among tertiary care hospitals, ${ }^{1}$ is in our opinion attributable

Table 1. Risk factors associated with candidemia.

\begin{tabular}{|c|c|c|}
\hline Risk factor & $\begin{array}{c}\text { Number of } \\
\text { patients }\end{array}$ & $\%$ \\
\hline Antibiotic therapy & 44 & 91.7 \\
\hline Parenteral nutrition & 39 & 81.2 \\
\hline Age $\geq 65$ years & 36 & 75.0 \\
\hline Male gender & 29 & 60.4 \\
\hline Central venous catheter* & 27 & 56.2 \\
\hline Age $\geq 75$ years & 26 & 54.2 \\
\hline Urinary catheter & 20 & 41.7 \\
\hline Solid tumor & 17 & 35.4 \\
\hline Corticosteroid therapy & 16 & 33.3 \\
\hline Surgery $<6$ months & 10 & 20.8 \\
\hline Provenience from other hospital wards & 10 & 20.8 \\
\hline Diabetes mellitus & 9 & 18.7 \\
\hline Abdominal surgery $<6$ months & 8 & 16.7 \\
\hline Cardiovascular disease & 7 & 14.6 \\
\hline Emergency ward $<1$ months & 6 & 12.5 \\
\hline Drainage $^{\circ}$ & 5 & 10.4 \\
\hline Dialysis & 4 & 8.3 \\
\hline Antifungal prophilaxis ${ }^{\#}$ & 4 & 8.3 \\
\hline Chemotherapy & 4 & 8.3 \\
\hline Mechanic ventilation & 4 & 8.3 \\
\hline Hematologic malignancies & 3 & 6.2 \\
\hline Tracheostomy & 3 & 6.2 \\
\hline Radiotherapy & 2 & 4.2 \\
\hline Bone marrow transplant & 1 & 2.1 \\
\hline Neutropenia $<1000 / \mu \mathrm{L}$ & 1 & 2.1 \\
\hline
\end{tabular}

Table 2. Distribution of Candida species.

\begin{tabular}{lccccc}
\hline Candida spp. & Latisana & Palmanova & S. Daniele & Tolmezzo & Total no. (\%) \\
\hline C. albicans & 6 & 6 & 10 & 7 & $29(60.4)$ \\
\hline C. parapsilosis & 2 & 2 & & $6^{*}$ & $10(20.8)$ \\
\hline C. tropicalis & 2 & 1 & 1 & 1 & $5(10.4)$ \\
\hline C. glabrata & 1 & - & 2 & - & $3(6.2)$ \\
\hline C. krusei & - & 1 & - & 14 & $1(2.1)$ \\
\hline Total & 11 & 10 & 13 & & 48 \\
\hline
\end{tabular}

* $42.9 \%$ of Tomezzo candidemias. 
Table 3. Antifungal susceptibility-test results.

\begin{tabular}{|c|c|c|c|}
\hline Candida spp. & Antifungal agent & No. isolates tested & $\begin{array}{l}\text { No. (\%) of resistant or } \\
\text { DDS isolates }\end{array}$ \\
\hline \multirow[t]{4}{*}{ C. albicans (29) } & Amphotericin B & 19 & 0 \\
\hline & Fluconazole & 19 & $2(10.5)$ \\
\hline & Voriconazole & 19 & $3(15.8)$ \\
\hline & Caspofungin & 11 & 0 \\
\hline \multirow[t]{4}{*}{ C. parapsilosis (10) } & Amphotericin B & 8 & 0 \\
\hline & Fluconazole & 8 & 0 \\
\hline & Voriconazole & 8 & 0 \\
\hline & Caspofungin & 1 & 0 \\
\hline \multirow[t]{4}{*}{ C. tropicalis (5) } & Amphotericin B & 4 & 0 \\
\hline & Fluconazole & 4 & 0 \\
\hline & Voriconazole & 4 & 0 \\
\hline & Caspofungin & 1 & 0 \\
\hline \multirow[t]{4}{*}{ C. glabrata (3) } & Amphotericin B & 3 & 0 \\
\hline & Fluconazole & 3 & $2(66.7)$ \\
\hline & Voriconazole & 3 & $1(33.3)$ \\
\hline & Caspofungin & 2 & 0 \\
\hline \multirow[t]{4}{*}{ C. krusei (1) } & Amphotericin B & 1 & 0 \\
\hline & Fluconazole & 1 & $1(100.0)$ \\
\hline & Voriconazole & 1 & 0 \\
\hline & Caspofungin & - & - \\
\hline \multirow[t]{4}{*}{ All Candida spp. (48) } & Amphotericin B & 35 & 0 \\
\hline & Fluconazole & 35 & $5(14.3)$ \\
\hline & Voriconazole & 35 & $4(11.4)$ \\
\hline & Caspofungin & 15 & 0 \\
\hline
\end{tabular}

DDS, dose-dependent sensitivity.

to a casual association with the most frequent diseases affecting the population admitted to IMW.

As far as the previously reported shift in the pathogenesis of candidemia towards a non-albicans etiology, ${ }^{18-21}$ we confirmed more recent data on the prevalence of Candida species: ${ }^{1,16}$ C. albicans still accounts for the majority of cases; $C$. parapsilosis is the second most common strain (maybe due to increasing use of parenteral nutrition and vascular catheters, as previously suggested) $;{ }^{18}$ C. tropicalis has established as the third more frequent species, as previously reported in IMW and among elderly people; ${ }^{16,22}$ frequency of $C$. glabrata and C. krusei confirmed to be low. The particularly high incidence of $C$. parapsilosis in Tolmezzo hospital (42.9\% of cases of this hospital), underlines the need for knowledge of local epidemiology, since this data might be useful when planning the best therapeutic strategy.

Our population of fluconazole-resistant or dose-dependent sensitivity (DDS) isolates was higher (14.3\%) than that reported in Italy-Spain (5.1\%), Europe (6.3\%) and North-America $(6.6 \%),{ }^{1,23,24}$ as it was the proportion of voriconazole-resistant or DDS isolates $(11.4 \%)$ when compared to the rate reported in Italy-Spain $(<3 \%)$. No resistance was found to amphotericin $\mathrm{B}$ and caspofungin; the low number of tests performed (in particular against caspofungin) does not allow to draw any conclusions, but this last molecule has already proved to be of particular efficacy both in the Italy-Spain report, ${ }^{1}$ and in a previous Italian study. ${ }^{25}$

Moreover it must be underlined that a great variability of antifungal agents tested over years and laboratories was observed, with no tests at all performed in 2010 in one laboratory and few in another, and differences in the methods used. In Table 3 only the antifungal agents suggested by the 2012 European Society of Clinical Microbiology and Infectious Diseases (ESCMID) guidelines for the therapy of candidemia are reported; nevertheless 29 isolates were tested also against flucytosine, 21 against itraconazole, 10 against ketoconazole and 9 against posaconazole.

In regards to the high mortality rate observed (41.7\%), this data confirms those recently reported: in fact it was similar to that found in the Barcelona area $(44 \%),{ }^{26}$ and in five hospitals of Italy and Spain (where the mortality - $45.9 \%$ - in IMW was second 
only to hemato-oncology and Intensive Care Unit wards), ${ }^{1}$ and only slightly inferior to that detected in an Italian tertiary care hospital (50.4\%). ${ }^{16}$ Age of internal medicine patients and the presence of multiple comorbidities may explain this poor outcome.

Our study has a number of limitations that should be taken in mind when interpreting the results, with the limited number of cases recorded being the most important. This data does not allow to generalize our observations to all community hospitals and may not be representative of all Italian regions. Nevertheless it shows that in community hospitals the great majority of BSI from Candida are recorded in IMW; that candida species distribution is similar to that of tertiary care hospitals, but local epidemiology can be different and must be known; that patient characteristics are also similar, with older age, comorbidities, concomitant therapies and presence of indwelling devices playing the major role as risk factors; and also that among smaller hospitals crude mortality maintains its high incidence. The confirmed resistance to azoles in part of the cases, opens the field to careful considerations on which would be the best therapeutic approach.

\section{References}

1. Bassetti M, Merelli M, Righi E, et al. Epidemiology, species distribution, antifungal susceptibility, and outcome of candidemia across five sites in Italy and Spain. J Clin Microbiol 2013;51:4167-72.

2. Wisplinghoff H, Bischoff T, Tallent SM, et al. Nosocomial bloodstream infections in US hospitals: analysis of 24,179 cases from a prospective nationwide surveillance study. Clin Infect Dis 2004;39:309-17.

3. Marchetti O, Bille J, Fluckiger U, et al. Fungal Infection Network of Switzerland. Epidemiology of candidaemia in Swiss tertiary care Hospitals: secular trends 19912000. Clin Infect Dis 2004;38:311-20.

4. Zaoutis TE, Argon J, Chu J, et al. The epidemiology and attributable outcomes of candidemia in adults and children hospitalized in the United States: a propensity analysis. Clin Infect Dis 2005;41:1232-9.

5. Leroy O, Gangneux JP, Montravers P, et al. Epidemiology, management, and risk factors for death of invasive Candida infections in critical care: a multicenter, prospective, observational study in France (2005-2006). Crit Care Med 2009;37:1612-8.

6. Horn DL, Neofytos D, Anaissie EJ, et al. Epidemiology and outcomes of candidemia in 2019 patients: data from the prospective antifungal therapy alliance registry. Clin Infect Dis 2009;48:1695-703.

7. Bassetti M, Taramasso L, Nicco E, et al. Epidemiology, species distribution, antifungal susceptibility and outcome of nosocomial candidemia in a tertiary care hospital in Italy. PLoS One 2011;6:e24198.

8. Kett DH, Azoulay E, Echeverria PM, et al. Candida bloodstream infections in intensive care units: analysis of the extended prevalence of infection in intensive care unit study. Crit Care Med 2011;39:665-70.
9. De Rosa FG, Trecarichi EM, Montrucchio C, et al. Mortality in patients with early- or late-onset candidaemia. J Antimicrob Chemother 2013;68:927-35.

10. Viscoli C, Girmenia C, Marinus A, et al. Candidemia in cancer patients: a prospective, multicenter surveillance study by the Invasive Fungal Infection Group (IFIG) of the European Organization for Research and Treatment of Cancer (EORTC). Clin lnfect Dis 1999;28:1071-9.

11. Bassetti M, Righi E, Costa A, et al. Epidemiological trends in nosocomial candidemia in intensive care. BMC Infect Dis 2006;6:21.

12. Nguyen MH, Peacock JE Jr, Morris AJ, et al. The changing face of Candidemia: emergence of non-Candida albicans species and antifungal resistance. Am J Med 1996;100:617-23.

13. Rocco TR, Reinsert SE, Simms HH. Effects of fluconazole administration in critically ill patients: analysis of bacterial and fungal resistance. Arch Surg 2000; 135:160-5.

14. Bassetti M, Ansaldi F, Nicolini L, et al. Incidence of candidaemia and relationship with fluconazole use in an intensive care unit. J Antimicrob Chemother 2009;64:625-9.

15. Luzzati R, Amalfitano G, Lazzarini L, et al. Nosocomial candidemia in non-neutropenic patients at an Italian tertiary care hospital. Eur J Clin Microbiol Infect Dis 2000;19:602-7.

16. Bassetti M, Molinari MP, Mussap M, et al. Candidaemia in internal medicine departments: the burden of a rising problem. Clin Microbiol Infect 2013;19:281-4.

17. Guimaraes T, Nucci M, Mendonca JS, et al. Epidemiology and predictors of a poor outcome in elderly patients with candidemia. Int J Infect Dis 2012;16:442-7.

18. Clark TA, Slavinski SA, Morgan J, et al. Epidemiologic and molecular characterization of an outbreak of Candida parapsilosis bloodstream infections in a community hospital. J Clin Microbiol 2004;42:4468-72.

19. Lin MY, Carmeli Y, Zumsteg J, et al. Prior antimicrobial therapy and risk for hospital acquired Candida glabrata and Candida krusei fungemia: a case-case-control study. Antimicrob. Agents Chemother 2005;49:4555-60.

20. Pappas PG, Rex JH, Lee J, et al. A prospective observational study of candidemia: epidemiology, therapy, and influences on mortality in hospitalized adult and pediatric patients. Clin Infect Dis 2009;37:634-43.

21. Pfaller MA, Messer SA, Moet GJ, et al. Candida bloodstream infections: comparison of species distribution and resistance to echinocandin and azole antifungal agents in Intensive Care Unit (ICU) and non-ICU settings in the SENTRY Antimicrobial Surveillance Program (20082009). Int J Antimicrob Agents 2011;38:65-9.

22. Nucci M, Queiroz-Telles F, Alvarado-Matute T, et al. Epidemiology of candidemia in Latin America: a laboratory-based survey. PLoS One 2013;8:e59373.

23. Tortorano AM, Kibbler C, Peman J, et al. Candidaemia in Europe: epidemiology and resistance. Int J Antimicrob Agents 2006;27:359-66.

24. Messer SA, Moet GJ, Kirvy JT, Jones RN. Activity of contemporary antifungal agents, including the novel echinocandin anidulafungin, tested against Candida spp., Cryptococcus spp., and Aspergillus spp.: report from the SENTRY antimicrobial surveillance program (2006 to 2007). J Clin. Microbiol 2009;47:1942-6.

25. Bassetti M, Trecarichi EM, Righi E, et al. Incidence, risk 
factors, and predictors of outcome of candidemia. Survey in 2 Italian university hospitals. Diagn Microbiol Infect Dis 2007;58:325-31.

26. Almirante B, Rodriguez D, Park BJ, et al. Epidemiology and predictors of mortality in cases of Candida bloodstream infection: results from population-based surveillance, Barcelona, Spain, from 2002 to 2003. J Clin Microbiol 2005;43:1829-35.

\section{APPENDIX}

Maurizio Ruscio and Fabrizio Pistola (Microbiology service, General Hospital, San Daniele del Friuli, UD); Nicola Bizzaro, Sonia Merluzzi and Fiorenza Pesente (Microbiology service, Hospitals of Tolmezzo and Gemona, UD); Silvia De Montis and Francesca Sirianni (Microbiology service, Hospitals of Latisana and Palmanova, UD). 